\title{
Cuidado e acompanhamento do lactente de alto risco no primeiro ano de vida.
}

\section{Care and follow-up of the high-risk infant in the first year of life.}

\author{
Giselly Araujo Santos' $\bullet$ Josiel Elisandro Werle ${ }^{2} \bullet$ Maria Antonia Ramos Costa ${ }^{3}$ Dandara Novakowski Spigolon $^{4}$ \\ Rodrigo Guimarães dos Santos Almeida ${ }^{5}$ Elen Ferraz Testo ${ }^{6}$
}

\begin{abstract}
RESUMO
Objetivo: conhecer a percepção de familiares quanto ao cuidado e acompanhamento ao lactente de alto risco, pelos profissionais de saúde ao longo do primeiro ano de vida. Materiais e Método: estudo de natureza qualitativa, realizado com sete cuidadores familiares de lactentes estratificadas como alto risco, em um município do interior do Brasil. Os dados foram coletados por meio de entrevista audiogravadas no domicilio, no período de agosto a setembro de 2018. Posteriormente a transcrição, as falas foram submetidas a análise modalidade temática. Resultados: Os participantes apontaram que as orientações oferecidas no momento da alta, favoreceram a insegurança frente as ações de cuidados básicos. Ademais, o acompanhamento na Atenção Primária a Saúde foi referido como fator facilitador do cuidado, embora a visita domiciliar tenha se apresentado como uma lacuna de atuação dos profissionais. Conclusão: $\bigcirc$ cuidado ao lactente de alto risco trás desafios para a família e a necessidade da oferta de informações quanto a realização de cuidados básicos e a necessidade de acompanhamento pelos profissionais de saúde.
\end{abstract}

Palavras chave: Lactente; Cuidado do Lactente; Relações Familiares; Enfermagem Pediátrica

\begin{abstract}
Objective: Get to know the family members' perception of care and monitoring of high-risk infants realized by health professionals during the newborn's first year of life. Materials and Methods: a qualitative study, realized with seven high-risk infant's caregivers in a Brazilian interior town. The data were collected through audio-recorded home interviews, from August to September 2018. After the transcription, the speeches were subjected to thematic modality analysis. Results: Participants pointed out that the guidelines offered at the time of discharge, favored the insecurity regarding basic attention actions. Furthermore, the follow-up in Primary Health Care was referred to as a facilitating factor of care, although home visiting was presented as a gap in professional practice. Conclusion: The high-risk infant care brings challenges to the family and the necessity to provide knowledge in terms of basic care and the need for health professionals monitoring.
\end{abstract}

Key words: Infant; Infant Care; Family Relations; Pediatric Nursing 


\section{INTRODUÇÃO}

A mortalidade infantil, caracterizada pela ocorrência de óbitos no primeiro ano de vida, constitui um indicador social e econômico. No ano de 2017 o Brasil apresentou uma taxa de mortalidade infantil de $12,8 /$ mil nascidos vivos. Por sua vez, no mesmo período a probabilidade de um recém-nascido não completar um ano de vida foi de $8,9 \%$ no estado do Paraná ${ }^{(1)}$.

Nesse contexto, torna-se premente $\circ$ desenvolvimento de ações intra e inter setoriais para prevenção da mortalidade infantil, uma vez que os fatores causais estão relacionados com melhorias das condições socioeconômicas, do incentivo a escolaridade e da acessibilidade aos serviços e recursos de saúde. No tocante das ações específicas do setor saúde destaca-se a importância das estratégias de monitoramento do estado nutricional das crianças, do aumento da cobertura de rede de esgoto e água tratada, do planejamento familiar, da adequada atenção ao pré-natal, parto e puerpério e do início precoce ao tratamento de possíveis morbidades ${ }^{(2)}$.

Logo, com a intenção de reduzir as taxas de morbimortalidade na infância, o Ministério da Saúde tem investido em políticas públicas. Para tanto, iniciou-se a implementação de ações que auxiliassem na organização e funcionamento dos serviços de saúde em rede, de modo a contribuir para um melhor acesso e qualidade, integralidade, resolutividade e humanização da assistência ${ }^{(3)}$.

Dentre as redes prioritárias destaca-se a Rede Cegonha, estabelecida pela portaria $n^{\circ} 1459 / 201$ I, que tem como objetivo o cuidado integral e de qualidade à muIher e a criança. Um dos componentes dessa Rede, é a estratificação de risco do recém-nascido ao nascer (risco habitual, intermediário e alto risco) ${ }^{(3)}$. Constituem critérios para estratificação de alto risco: a prematuridade, asfixia grave (APGAR menor que 7 no quinto minuto de vida), baixo peso ao nascer, desnutrição grave, crescimento e/ou desenvolvimento inadequados intrauterino, presença de doenças de transmissão vertical e triagem neonatal positiva. Cabe salientar que a finalidade da estratificação de risco é organizar o fluxo de atendimento e a frequência de acordo com as diferentes necessidades, favorecendo o princípio da equidade ${ }^{(4)}$.

Nesse contexto, considerando as particularidades de cada lactente/família, o acompanhamento pela equipe de saúde contribui para o crescimento, desenvolvimento saudável e redução dos fatores de risco para mortalidade infantil, em especial para os lactentes de alto risco. Diante disso o enfermeiro desempenha ações de cuidado nas diferentes pontas da Rede de Atenção à Saúde (RAS) por meio da consulta de enfermagem, visitas domiciliares e atividades de educação em saúde ${ }^{(5)}$.

No entanto é necessário uma comunicação efetiva entre os serviços que compõem a rede e o desenvol- vimento de fluxos de atendimento do lactente, a fim de que o cuidado e acompanhamento seja longitudinal, compartilhado, equânime e integral ${ }^{(6)}$. Propicia assim qualidade na assistência no processo de desospitalização.

Frente a esse contexto questiona-se: como ocorre o cuidado e acompanhamento do lactente de alto risco ao longo do primeiro ano de vida nos diferentes pontos da Rede de Atenção à Saúde? E para responder esse questionamento o presente estudo tem como objetivo conhecer a percepção de familiares quanto ao cuidado e acompanhamento ao lactente de alto risco, pelos profissionais de saúde ao longo do primeiro ano de vida.

\section{MÉTODOS}

Estudo exploratório de natureza qualitativa, realizado com familiares, de lactentes de alto risco, residentes em um município de médio porte, localizado na região noroeste do Paraná. Esse possui 81.590 habitantes, de acordo com o último censo, e é sede de uma das 22 Regionais de Saúde do Paraná, que agrega 20 municípios. $\mathrm{Na}$ área da saúde para acompanhamento do lactente de alto risco, por ocasião do estudo, possuía para atendimento, 17 UBS's, todas com ESF (total de 24 equipes), Pronto Atendimento Municipal, um hospital privado conveniado ao Sistema Único de Saúde (SUS), Centro Regional de Especialidades (CRE), Sistema Integrado de Atenção à Saúde (SINAS) e Unidade de Atendimento Móvel.

Os participantes foram selecionados adotando-se os seguintes critérios de inclusão: ser o principal familiar cuidador do lactente, ter acompanhado o primeiro ano de vida e ter idade igual ou superior a 18 anos. Por sua vez, foram excluídos os familiares de lactentes que foram a óbito e/ou mudaram de cidade.

Após aprovação do Comitê de Ética em Pesquisa (Parecer $n^{\circ}$ 2.798.410), foi solicitado a coordenação do Programa Saúde da mulher, do município em estudo, uma lista com o nome, endereço e UBS de referência dos lactentes que nasceram no ano de 2017 e foram estratificadas como alto risco (34). Posteriormente, foi selecionada a UBS com o maior número dessas lactentes (I5).Após contato inicial com o Agente Comunitário de Saúde (ACS) procedeu-se a realização da visita domiciliar, pelo pesquisador principal e o ACS, para busca ativa desses lactentes, convidando o familiar para participação na pesquisa e posterior realização da entrevista, que teve duração média de 25 minutos.

Os dados foram coletados no período de agosto a setembro de 2018, por meio de entrevista individual gravada utilizando-se a seguinte questão norteadora: Fale sobre o cuidado e acompanhamento do lactente, pela equipe de saúde, ao longo do primeiro ano de vida. $A$ busca por informações ocorreu até o momento em que a ocorrência de significados foi convergente e o objetivo da pesquisa foi alcançado ${ }^{(7)}$. 
As entrevistas foram transcritas na íntegra no mesmo dia em que foram realizadas, e submetidas à análise de conteúdo, modalidade temática, seguindo as etapas de pré-análise, exploração do material, levantamento dos resultados e interpretação. Na pré-análise realizou-se a leitura das falas e o levantamento dos pontos chaves, de acordo com o objetivo proposto. Posteriormente, procedeu-se a codificação dos dados e finalmente a categorização, por meio da junção entre os elementos que se assemelham, originando duas categorias temáticas ${ }^{(8)}$.

Com vistas à garantia do anonimato dos participantes, as falas foram identificadas com a letra P de participante, seguida pelo número arábico, indicativo da ordem de realização das entrevistas e o vínculo familiar. Ex: (PI, mãe).

\section{RESULTADOS}

Participaram do estudo sete familiares com média de 30 anos (mínimo 22 e máximo 59 anos), sendo seis mães e uma avó materna.

Observou-se com relação ao acompanhamento dos lactentes de alto risco, que apenas uma delas possuía atraso vacinal em decorrência de hospitalização, todas possuíam registro de acompanhamento ao longo do primeiro ano de vida. Entretanto, nenhum dos gráficos de crescimento e desenvolvimento estavam completamente preenchidos.

\section{Fatores que favorecem o cuidado familiar ao bebê de alto risco: do hospital ao domicílio}

Observou-se $\circ$ desconhecimento/incompreensão dos familiares com relação aos fatores que tornavam o lactente como de alto risco após o nascimento:

$\mathrm{Na}$ verdade, ela não era de alto risco ela só nasceu baixo peso. (PI, mãe).

O alto risco deve ser porque eu fumo [...]. (P7, mãe).

Disseram que ele era alto risco porque era prematuro[...] mas isso é alto risco? (P2, mãe).

E quando cientes da estratificação de alto risco, os familiares referiram dificuldades e inseguranças na execução de ações de cuidados básicos:

Tinha medo de dar banho, dela não ganhar peso e precisar ficar internada [...]medo de mexer com ela[...]. (PI, mãe).

Eu tinha medo de dar banho nele, quando soube que ele era de risco fiquei sem saber como cuidar[...] depois meu peito rachou, eu chorava na hora de dar mama, tive que dar $x x x$ (fórmula de leite infantil) para ele, daí ele ficou ressecado não fazia cocô, teve cólica, chorava um monte a noite. (P4, mãe).

Para minha família foi bem dificil, ele era muito pequeno, e até hoje ele é menor que as outras crianças, já tenho outra filha, mas esse foi mais dificil para eu cuidar[...] parecida que eu não sabia. Eu e meu marido não dormíamos com medo de acontecer alguma coisa com ele. (P5, mãe).
Entretanto alguns familiares apontaram que as orientações ofertadas pela equipe de saúde no momento da alta hospitalar favoreceram o enfrentamento das dificuldades e dúvidas na realização do cuidado no domicílio:

Eles me explicaram como que eu tinha que dar o banho e dar de mamar, isso me ajudou muito porque sentia medo de mexer nele por ser de risco. Agendaram uma consulta com a pediatra para eu levar uma semana depois da alta e disseram que ela teria que realizar esse acompanhamento por ser de alto risco. (P6, mãe).

Me disseram que eu tinha que ir na pediatra e depois no postinho, me falaram que eu tinha que cuidar da alimentação porque se não ganhasse peso podia atrapalhar o crescimento. (PI, mãe).

Tive bastante orientação, falaram sobre ele ser prematuro, ele teve hérnia e me ensinaram a cuidar, me ensinaram a dar banho e a sugar o seio. (P2, mãe).

Me falaram que eu tinha que ter muito cuidado com ele porque era prematuro e também iria ser especial, como ele nasceu muito pequeno, eu tinha que cuidar com muitas visitas mandar elas lavarem as mãos, cuidado com o banho, tinha que dar o banho em um horário mais quente, e cuidar para ele não ficar muito tempo sem mamar. (P4, mãe).

Destacaram também a importância da contra referência realizada na maternidade para os outros pontos da rede de atenção à saúde:

Me mandaram ir no postinho para acompanhar o crescimento e desenvolvimento...ele foi consultado com o médico do posto e a enfermeira e também passou no centro de especialidades com a pediatra três vezes [...]. (P5, mãe).

Tem uma moça que vai no quarto entregar a carteirinha dos bebês e ela me falou que iria agendar uma consulta com a pediatra. E quando eu saí do hospital me entregaram um papel com a consulta agendada, também falaram para eu ir no posto de saúde para fazer as primeiras vacinas, e tive que voltar uma semana depois para refazer o teste da orelhinha (P7, mãe).

Do mesmo modo, a rede de apoio representada pelo envolvimento de outros familiares no cuidado do lactente ao longo do primeiro ano de vida também foi considerada como fator de influência positiva:

Ela era muito pequena, as roupas ficaram grandes e tivemos que comprar mais, e meu marido usa drogas, então sofri muito, a minha sogra que me ajudou bastante no cuidado. (PI, mãe).

A mãe não ajudou a cuidar... passamos por várias dificuldades mas quem pude contar foi com minha filha mais nova. (P3, avó materna).

Então ele era muito pequeno, tudo tinha que ser feito com muito cuidado, mais graças a Deus consegui cuidar bem dele, meu marido me ajudou muito, revezávamos as noites, ele era muito quietinho. (P6, mãe). 
Acompanhamento do lactente de alto risco nos diferentes pontos da Rede de Atenção à Saúde

Os familiares referiram que o acompanhamento do lactente ao longo do primeiro ano de vida foi realizado na maioria das vezes na UBS na consulta de puericultura:

Ela fez acompanhamento no postinho a maioria das vezes com a enfermeira que pesava e media e oferecia algumas informações. Passava uma vez com o médico e outra com a enfermeira. (PI, mãe).

Fez as consultas no postinho de puericultura com a enfermeira, participou do programa de pesagem para poder receber o leite e também foi no dentista. (P4, mãe).

Todo mês tinha consulta marcada no postinho ou com a médica ou com a enfermeira, olhavam a carteirinha para ver as vacinas, pesava, media e perguntava como estava de saúde. (P3, avó materna).

Por vezes, os familiares referiram alguns atendimentos em outros pontos da rede de atenção, por exemplo, no centro de especialidades com a pediatra:

Fez acompanhamento com a pediatra no centro de especialidades, me senti muito segura por ser especialista. Como era de alto risco teve direito a passar várias vezes por ela. (P2, mãe).

Pediatra e com o oftalmologista acompanhou também, porque o canal da lágrima é obstruído. (P3, avó materna).

Além do postinho ele passava com a pediatra todo mês também isso me deixou tranquila[...] foi cuidada por muita gente da saúde. (P6, mãe).

Entretanto, apontaram a ausência ou insuficiência de visita domiciliar, como um fator que não favoreceu o cuidado e acompanhamento da criança:

Não recebia visita, as ACS passaram na minha sogra e perguntaram se estava tudo bem comigo e com o bebê mas isso foi só no primeiro mês (eu morava no fundo). (PI, mãe).

Eles poderiam vir mais vezes aqui na minha casa. Meu peito doeu muito pra dar mama e eu não conseguia [...] desisti de amamentar e estava sem carro para ir até o postinho. (PI, mãe).

A bebê ficou muito ressecada por causa da fórmula e não fazia cocô, o farmacêutico amigo da minha sogra que passou remédio, acho que faltou um pouco de acompanhamento em casa[...].no começo é muito dificil a gente ir até o posto. Descobriram que o bebê teve isso quando eu fui até lá e contei [...]um mês depois. (PI, mãe).

Precisa de visitas domiciliares, porque a mãe da bebê não cuida direito e acaba não escutando as nossas orientações, e se tivesse vindo um profissional de saúde seria mais fácil dela aceitar. (P3, avó materna).

\section{DISCUSSÃO}

Conhecer como ocorre o cuidado e acompanhamento do lactente de alto risco e sua família pela equipe de saúde nos diferentes pontos de atenção da RAS ao lon- go do primeiro ano de vida possibilita a identificação de fatores que influenciam positiva e negativamente nesse processo. Desse modo, torna-se possível a elaboração de estratégias de intervenção que favoreçam o enfrentamento de dificuldades e a segurança dos familiares frente ao processo de cuidado. Por meio da estratificação de risco é possível desenvolver um cuidado que atenda as especificidades de cada lactente e concentrar maiores ações para aqueles que possuem maior probabilidade de adoecer e morrer ${ }^{(4)}$.

Para tanto, é necessário que a equipe de saúde esclareça para a família desses bebês, os fatores que as estratificam como alto risco para que esses possam realizar um cuidado compartilhado com a equipe de saúde, de forma segura e capaz de identificar precocemente possíveis agravos. Por vezes, os desconhecimentos desses fatores como identificado no presente estudo podem influenciar negativamente na condução do cuidado, uma vez que o familiar pode não relacionar o problema como o baixo peso, por exemplo, como fator influente no crescimento e desenvolvimento.

Ademias, o desconhecimento do risco também pode resultar em insegurança pelos familiares para realização de cuidados básicos, uma vez que estes idealizam a maternidade e o nascimento sem complicações. Desse modo, ao se deparar com um cenário diferente do esperado, experienciam a frustação e sentimentos de medo e insegurança. Assim, a atuação da equipe de saúde deve favorecer o conhecimento da família, possibilita sanar as dúvidas o que contribui para promoção de autonomia e segurança no cuidado a esses bebês ${ }^{(9)}$.

Por vezes, quando cientes da existência de fatores de risco como, por exemplo, o baixo peso ou a prematuridade, os familiares apresentam maior temor em acontecer algum problema com o lactente na residência ${ }^{(10)}$. Observou-se que umas das estratégias que auxiliaram no enfrentamento da insegurança frente ao cuidado do bebê de alto risco pelos familiares foram às orientações oferecidas pelos profissionais de saúde no momento da alta hospitalar.

Nesse sentido, oportunizar espaço de diálogo com a família para conhecer as principais angústias pode auxiliar na compreensão e segurança para realização de cuidados básicos, como o banho. Estudo realizado em uma UTI neonatal localizado no norte do Paraná com os pais, apontou que as orientações sobre prematuridade são essências, a escuta e apoio oferecido aos familiares contribuem para redução dos medos e dúvidas quanto ao cuidado com os bebês. Os pais foram permitidos a participarem do cuidado como troca de fraldas, realização do banho, alimentação, atenção durante a noite, hábitos de higiene e prevenção de doenças, tornando-os preparados para a alta hospitalar e cuidados no domicilio(II). 
Aliado a isso, torna-se essencial o acolhimento da mãe e do bebê no domicílio pela equipe da Atenção Primária à Saúde, o qual é oportunizado pela contra referência realizada pela maternidade à UBS correspondente. Assim, ressalta-se a necessidade de encaminhas a mãe e o bebê com os dados sobre sua permanência no hospital, resumo da alta e a principalmente as intercorrências com vistas a possibilitar a continuidade do cuidado. $O$ instrumento facilitador para essa referência é a ficha do recém-nascido de risco, onde contém informações como dados da mãe (idade, endereço, bairro, etc.), dados do nascimento (idade gestacional, tipo de parto, APGAR, anomalias, etc.), critérios de inclusão e dados da alta hospitalar. Esse instrumento deve ser preenchido corretamente e enviado a UBS de referência, facilitando a busca ativa desses indivíduos pelos membros da estratégia de Saúde da Família ${ }^{(12)}$.

A RAS tem como um dos objetivos acolher a mãe e - lactente nos diferentes períodos e contribuir para a realização do cuidado integral. Entretanto, um estudo realizado com instituições de apoio a crianças com necessidades especiais de saúde em Ribeirão Preto (SP) apontou que a dificuldade de comunicação entre os diferentes pontos da RAS, compromete a qualidade do cuidado ${ }^{(13)}$. Nesse sentido, o estabelecimento de fluxos de serviço entre os diferentes pontos da rede, tornam-se necessários a fim de amenizar as lacunas de comunicação(14).

Outro fator positivo para o cuidado e acompanhamento do lactente destacada pelos familiares é a rede de apoio estabelecida que independente do vínculo ofereça suporte ao cuidador principal, que por vezes, é a mãe. Nesse sentido, a redução de reinternações desnecessárias, um melhor desenvolvimento e crescimento do lactente é influenciado pelo cuidado que esse recebe da rede de apoio familiar, o que também favorece a melhor qualidade de vida para os pais e/ou cuidadores ${ }^{(9)}$.

Estudo ${ }^{(15)}$ demonstrou discreta redução de óbitos por causas evitáveis no período neonatal embora, em outras classificações da mortalidade infantil não houve redução significativa da mortalidade infantil após implantação Rede Mãe Paranaense (RMP). Em decorrência da rede temática ser relativamente recente o processo de mensurar os indicadores deve ser pensado a longo prazo.

Destaca-se ainda que para haver redução dos óbitos pós-neonatais, é fundamental desfrutar de ações que visam promoção e prevenção, possibilite acesso a serviços de saúde, investimentos nas capacitações dos profissionais de saúde envolvidos, principalmente ao enfermeiro que atua na atenção básica, para que o mesmo possa ter um olhar atento para ações de cuidado, promovendo saúde e detecção precoce de doenças para diminuir sobremaneira indicadores de mortalidade infantil( ${ }^{(15)}$.

O acompanhamento pela equipe de saúde ao lactente de alto risco e sua família, ao longo do primeiro ano de vida, favorece a identificação precoce de alterações na condição de saúde do lactente e o estabelecimento de intervenções. Uma das estratégias utilizadas frequentemente é a puericultura que oportuniza o estreitamento de vínculo entre a equipe de saúde e família, proporcionando ações de promoção a saúde e informações quanto aos cuidados gerais. Entretanto, estudo realizado no município de Maringá (PR) com familiares de crianças que estavam inscritas em um programa de puericultura concluiu que a maior parte dos familiares entrevistados não possuíam conhecimento a respeito da importância desse acompanhamento(16).

O Ministério da Saúde propõe um calendário mínimo de sete consultas de puericultura no primeiro ano de vida, afim de oportunizar o acompanhamento do crescimento e desenvolvimento infantil. Esse calendário é seguido na grande maioria dos serviços de APS no Brasil, o qual articula as consultas médicas e de enfermagem. As orientações acerca das condições de saúde do lactente constituem configurações de cuidado e de continuidade a assistência ao recém-nascido com risco ${ }^{(17)}$.

O enfermeiro ao solicitar a caderneta da criança, procede o acompanhamento no gráfico de crescimento e desenvolvimento, anota dados sobre histórico familiar, informações sobre alimentação, exame clínico, condutas, bem como as orientações prestadas aos responsáveis ${ }^{(18)}$. Essa sistematização auxilia os profissionais de saúde no desenvolvimento do cuidado continuado ao lactente, o que facilita a identificação de alterações no seu padrão de desenvolvimento, harmonizando as orientações e direções necessárias para o seu atendimento nos diferentes pontos da RAS.

Ademais, vale considerar ainda a importância da busca ativa dos lactentes de alto risco pela equipe de saúde, uma vez que nem todos são assíduas ao acompanhamento do processo de crescimento e desenvolvimento. Para tanto, a visita domiciliar constitui uma estratégia que possibilita a identificação precoce de alterações e dificuldades vivenciadas no processo de cuidado pelos familiares ao lactente ${ }^{(17)}$.

Atinente a isso, estudo realizado em Unidades de Saúde da Família no interior da Bahia apontou que a enfermagem realiza visitas domiciliares com prioridade as pessoas que têm dificuldades de deslocamento para a UBS.Ademais, apontou que a prática de cuidado na visita domiciliar, tem pouco direcionamento para questões de promoção à saúde, pois há maior enfoque em aspectos biológicos, desconectando da historicidade e singularidade de cada ser humano. Assim, reitera-se a importância da visita domiciliar para busca ativa e cuidado ao lactente de alto risco, uma vez que nesse cenário o profissional de saúde tem a oportunidade de conhecer o meio familiar e 
identificar as fragilidades e potencialidades no processo do cuidado além de fortalecer a ligação entre a equipe e a família(19).

Vale destacar que os profissionais de saúde são importantes auxiliadores as mães e responsáveis em relação aos cuidados de saúde dos lactentes em ambiente domiciliar e estes podem ser oportunizados por meio da visita domiciliar ${ }^{(20)}$. Ressalta-se ainda, que a falta de visita domiciliar, é um problema no cuidado à saúde, visto que se identifica no contexto domiciliar as particularidades que influenciam no processo de saúde doença.

Destaca-se como limitação do estudo a inclusão de apenas uma UBS, uma vez que os aspectos sociodemográficos podem influenciar nos resultados encontrados. Entretanto, com os resultados pode-se apreender algumas lacunas existentes no processo de acompanhamento do lactente de alto risco ao longo do primeiro ano de vida, o que deve ser alvo de ações dos profissionais de saúde a fim de aprimorar a qualidade e a integralidade do cuidado prestado.

\section{CONCLUSÃO}

Os resultados deste estudo demonstraram que os familiares dos lactentes de alto risco percebem o desconhecimento/incompreensão e a insegurança como fatores influentes no processo de cuidado ao longo do primeiro ano de vida.Além disso, destacaram as estratégias que favorecem o cuidado como orientações recebidas no momento da alta hospitalar, a contra referência para UBS e os outros pontos de atenção e a rede de apoio.

Por outro lado, alguns participantes relataram que a ausência e/ou insuficiência de visita domiciliar pela equipe de saúde constitui um fator negativo para a identificação de necessidades no processo de cuidado à criança e vinculação à equipe de saúde. Diante disso destaca-se a necessidade dos profissionais de saúde, em especial aqueles que atuam na Atenção Primária, traçarem estratégias de acompanhamento à criança/ família, intercalando consultas na unidade e visitas domiciliares a fim de fortalecer o vínculo, identificar necessidades especificas e elaborar em conjunto o plano de cuidados.

Sendo assim, é notório que os achados deste estudo podem auxiliar o enfermeiro da ESF a repensar a organização do processo de trabalho da equipe, em especial no acompanhamento das crianças de alto risco. 


\section{REFERÊNCIAS}

I. IBGE.Tábua completa de mortalidade para o Brasil - 2017: Coordenação de Populações e Indicadores Sociais. 2017. [citado 2019 set. 07] Disponível em ftp://ftp.ibge.gov.br/ Tabuas_Completas_de_Mortalidade/Tabuas_Completas_ de_Mortalidade_2017/tabua_de_mortalidade_2017_analise.pdf.

2. Dias BAS, Neto ETDS, Andrade MAC. Classificação da Evitabilidade dos óbitos infantis: diferentes métodos, diferentes repercussões? [Internet] 2017; [citado 2018 març. 02]. Cad Saúde Pública; 33(5): e 00I259|6. Disponível em: http://www.scielo.br/scielo.php?script=sci_arttext\&pi$d=S 01023$ I | X20 I 7000504002\&lng=en\&nrm=iso\&tlng=pt

3. Ministério da Saúde (BR). Portaria $n^{\circ} 4279 / 2010$. Implantação das Redes de Atenção à Saúde. [Internet] 2010; [citado 2018 març. 10]. Disponível em: http://conselho. saude.gov.br/ultimas_noticias/20l l/img/07_jan_portaria4279_301210.pdf

4. Linha Guia/ Rede Mãe Paranaense. Caderno de Atenção Básica, crescimento e desenvolvimento infantil. Secretaria da Saúde do Estado do Paraná. [Internet] 20I4; [citado 2018 abr. 03] Disponível em: www.saude.pr.gov.br/File/ACS/linha_guia_versao_final.pdf

5. Aparecida MGM, Aparecida CMC, Dias SMM, Marques SAK. Avaliação do crescimento e desenvolvimento infantil na consulta de enfermagem. av.enferm. [Internet]. $2018 \mathrm{Abr}$ [citado 2019 Sep 08] ; 36( I ): 9-2I. Disponível em: http://www.scielo.org.co/scielo.php?script=sci_arttext\&pi$\mathrm{d}=\mathrm{SO} / 2 \mathrm{I}-$

6. Vieira CS, Lomba GDO, Costa MA, Braga PP, Gesteira ECR. Vivencia Discente e Docente Na Continuidade do Cuidado a Criança de Risco: Relato de Experiência. [Internet] 20I4; [citado 2018 abr. 20]. Disponível em: http://www.seer.ufsj. edu.br/index.php/recom/article/view/674

7. Pires AP. Amostragem e pesquisa qualitativa: ensaio teórico e metodológico. In: Poupart J, Deslauriers JP, Groulx LH, Lapemère A, Mayer R, Pires AP, organizadores. A pesquisa qualitativa: enfoques epistemológicos e metodológicos. Petrópolis: Editora Vozes; 2008. p. 154-2II

8. Bardin L. Análise de conteúdo. Tradução de Luís Antero Reto e Augusto Pinheiro. São Paulo: Edições 70, 201 I.

9. Oliveira MC, Locks MOH, Girondi JBR, Costa R. Método Canguru: percepção das mães que vivenciam a segunda etapa. [Internet]. 2015; [citado 2018 jul. 10] Disponível em: http://pesquisa.bvsalud.org/enfermagem/resource/pt/ lil-762257

10. Nascimento MLD, Pieszak GM, Arrué AM, Carvalho SORM. Crianças egressas de terapia intensiva neonatal: implicações para as redes sociais de cuidado. [Internet]. 2016; [citado 2018 abr. 2l]. Disponível em: http://www.periodicos.ufc.br/ rene/article/view/6207

I I.Zani AV, Silva TRD, Parada CMGDL. Os primeiros dias do fiIho prematuro em casa: discurso do sujeito coletivo. [Inter- net]. 2017; [citado ago. 02] Disponível em: http://pesquisa. bvsalud.org/enfermagem/resource/pt/biblio-877246

12. Ministério da Saúde(BR). Atenção à saúde do recém-nascido. Guia para os profissionais de saúde. Cuidados gerais. 2 ed. [Internet]. 20।2; [citado 2018 abr. 22] Disponível em: http://bvsms.saude.gov.br/bvs/publicacoes/atencao_saude_ recem_nascido_profissionais_vl.pdf

13. Astolpho MP, Okido ACC, Lima RAG. Rede de cuidados a crianças com necessidades especiais de saúde. [Internet]. 2014; [citado 2018 set. 08] Disponível em: http://pesquisa. bvsalud.org/enfermagem/resource/pt/mdl-24861063

14. Silva-Rodrigues FM, Bernardo CSG, Alvarenga WA, Janzen DC, Nascimento LC. Transição de cuidados para - domicílio na perspectiva de pais de filhos com leucemia. Rev Gaúcha Enferm. [Internet]. 2019 [citado 2019 set. 24];40:e20180238. Disponível em: https://doi. org/10.1590/1983-1447.2019.20180238

15. Netto A, Silva RMM, Santos MF,Tacla MTGM, Caldeira S, Brischiliari SCR. Mortalidade Infantil:avaliação do Programa Rede Mãe Paranaense em regional de saúde do Paraná. Cogitare Enfermagem [Internet]. 2017 Jan. 20 [citado em 2019 Abr. 4];22(I):8-20. Disponível em: http://dx.doi.org//0.5380/ce.v22il.47634.

16. Malaquias TDSM, Gaíva MAM, Higarashi IH. Percepção dos familiares de crianças sobre a consulta de puericultura na estratégia saúde da família. [Internet]. 2015; [citado 2018 jun. 20] Disponível em: http://pesquisa.bvsalud.org/enfermagem/resource/pt/mdl-26098804

17. Almeida AC, Mendes LC, Sad IR, Ramos EG, Fonseca VM, Peixoto MVM. Uso de instrumento de acompanhamento do crescimento e desenvolvimento da criança no Brasil Revisão sistemática de literatura. Rev. paul. pediatr. [Internet]. 2016 Mar [cited 2019 Sep 08]; 34(I): I22-|3|.Available from: http://www.scielo.br/scielo.php?script=sci_arttext\&pid=S0 I03-05822016000 I00 I 22\&lng=en.

18. Moura RS, Saraiva FJC, Santos RFM, Porfírio ABV, Leite GF, Felippu JSTM. Diagnostico de Enfermagem durante a Consulta de Puericultura: Relato de Experiência. Olhares Plurais. Rev. Eletrônica multidisciplinar, vol.2, $n^{\circ}$ 13, 2015.

19. Santos FPA, Acioli S, Rodrigues VP, Machado JC, Souza MS, Couto TA. Nurse care practices in the Family Health Strategy. Rev. Bras. Enferm. [Internet]. 2016 Dec [cited 2019 Sep 08]; 69(6): ||24-I|3|. Available from: http://www.scielo.br/scielo.php?script=sci_arttext\&pi$d=$ S0034-7 I 6720 I600060 I I 24\&lng=en.

20. Ribeiro BM dos SS, Silva VA, Teston EF, Hirai VHG, Souza $S R$, Curty MCR. Sentimentos de mães que passaram por situações de urgência e emergência com seus filhos em ambiente domiciliar. REAS [Internet]. 29nov.2018 [citado 8set.2019];II(I):e76. Available from: https://acervomais. com.br/index.php/saude/article/view/76.

Recebido: 2019-10-30

Aceito: 2020-02-03 